\title{
Análise sobre a sustentabilidade financeira para garantia do acesso integral aos medicamentos oncológicos
}

\author{
Analysis of financial sustainability to guarantee full access to cancer drugs \\ Análisis de sostenibilidad financiera para garantizar el pleno acceso a los medicamentos contra el \\ cáncer
}

\author{
José de Arimatea Rocha Filho \\ ORCID: https://orcid.org/0000-0003-2682-9995 \\ Universidade Federal de Pernambuco, Brasil \\ E-mail: arimatearf@gmail.com \\ Carolina Barbosa Brito da Matta \\ ORCID: https://orcid.org/0000-0002-1443-2029 \\ Universidade Federal de Pernambuco, Brasil \\ E-mail: carolinadamatta.hc@gmail.com \\ Thiago Douberin da Silva \\ ORCID: https://orcid.org/0000-0002-6925-6392 \\ Universidade Federal de Pernambuco, Brasil \\ E-mail: thiagodouberin@gmail.com \\ Doralice Conceição da Paz Neta \\ ORCID: https://orcid.org/0000-0001-5057-6460 \\ Universidade Federal de Pernambuco, Brasil \\ E-mail: pazdoralice@ gmail.com \\ Daniel Charles dos Santos Macêdo \\ ORCID: https://orcid.org/0000-0002-2356-5149 \\ Universidade Federal de Pernambuco, Brasil \\ E-mail: daniel.csmacedo@ufpe.br \\ Maira Galdino da Rocha Pitta \\ ORCID: https://orcid.org/0000-0001-5881-0338 \\ Universidade Federal de Pernambuco, Brasil \\ E-mail:mgrpitta@gmail.com \\ Antônio Guedes Correia Gondim Filho \\ ORCID: https://orcid.org/0000-0002-7084-8694 \\ Universidade Federal de Pernambuco, Brasil \\ E-mail: antonio.ebserh@gmail.com \\ Michelly Cristiny Pereira \\ ORCID: https://orcid.org/0000-0002-1672-8202 \\ Universidade Federal de Pernambuco, Brasil \\ E-mail: michelly.pereira@ufpe.br
}

\begin{abstract}
Resumo
A alta incidência dos casos de câncer tornou-o evidente problema de saúde pública em todo o mundo. Os gestores da rede pública no Brasil alegam que os recursos repassados não são suficientes para cobrir os custos com os quimioterápicos. Este trabalho trouxe um panorama do serviço ofertado pelo SUS na rede de atenção oncológica em Pernambuco e avaliou a sustentabilidade financeira para garantir o acesso aos medicamentos quimioterápicos em um hospital referência. Tratou-se de um estudo observacional, analítico e retrospectivo, realizado no período de 2015 a 2020. Avaliou-se o custo dos quimioterápicos para o câncer de mama e foram analisadas as incorporações de novos tratamentos pela CONITEC durante o mesmo período. Foram realizados 343.585 procedimentos quimioterápicos nos estabelecimentos avaliados e observou-se que o câncer de mama foi a neoplasia mais onerosa. Observou-se ainda que o financiamento dos tratamentos oncológicos se manteve estável, apesar da evolução inflacionária e epidemiológica, havendo prejuízo na incorporação de tecnologias. Verificou-se que os custos com quimioterápicos para câncer de mama comprometeram 31,23\% do valor pago pelo MS, e atualmente, com os reajustes do período, este passou a comprometer 100,75\%. Ao projetar os reajustes para os próximos anos, verificou-se que o orçamento estaria comprometido em 121,6\%, levando em conta o mesmo cenário inflacionário. Concluiu-se que a atual forma de financiamento do tratamento quimioterápico para o câncer de mama não se mostrou financeiramente sustentável no cenário avaliado e que se novos investimentos não forem aportados, os pacientes do SUS não serão beneficiados com as novas tecnologias de tratamento.
\end{abstract}

Palavras-chave: Câncer; Tratamento; SUS; Financiamento. 


\begin{abstract}
The high incidence of cancer cases has made it an evident public health problem worldwide. Public network managers in Brazil claim that the funds transferred are not sufficient to cover the costs of chemotherapy. This work provided an overview of the service offered by the SUS in the oncology care network in Pernambuco and evaluated the financial sustainability to ensure access to chemotherapy drugs in a reference hospital. This was an observational, analytical and retrospective study, carried out from 2015 to 2020. The cost of chemotherapy for breast cancer was evaluated and the incorporation of new treatments by CONITEC during the same period was analyzed. A total of 343,585 chemotherapy procedures were performed in the establishments evaluated and it was observed that breast cancer was the most costly neoplasm. It was also observed that the financing of cancer treatments remained stable, despite the inflationary and epidemiological evolution, with a loss in the incorporation of technologies. It was found that the costs of chemotherapy for breast cancer compromised $31.23 \%$ of the amount paid by the MS, and currently, with the readjustments in the period, this started to compromise $100.75 \%$. When projecting the readjustments for the coming years, it was found that the budget would be committed at $121.6 \%$, taking into account the same inflationary scenario. It was concluded that the current form of financing chemotherapy treatment for breast cancer did not prove to be financially sustainable in the evaluated scenario and that if new investments are not made, SUS patients will not benefit from new treatment technologies.
\end{abstract}

Keywords: Cancer; Treatment; SUS; Financing.

\title{
Resumen
}

La alta incidencia de casos de cáncer lo ha convertido en un evidente problema de salud pública en todo el mundo. Los administradores de redes públicas en Brasil afirman que los fondos transferidos no son suficientes para cubrir los costos de la quimioterapia. Este trabajo brindó una visión general del servicio que ofrece el SUS en la red de atención oncológica de Pernambuco y evaluó la sostenibilidad financiera para garantizar el acceso a los medicamentos de quimioterapia en un hospital de referencia. Se trata de un estudio observacional, analítico y retrospectivo, realizado entre 2015 y 2020. Se evaluó el costo de la quimioterapia para el cáncer de mama y se analizó la incorporación de nuevos tratamientos por parte del CONITEC durante el mismo período. Se realizaron un total de 343.585 procedimientos de quimioterapia en los establecimientos evaluados y se observó que el cáncer de mama fue la neoplasia más costosa. También se observó que el financiamiento de los tratamientos oncológicos se mantuvo estable, a pesar de la evolución inflacionaria y epidemiológica, con una pérdida en la incorporación de tecnologías. Se encontró que los costos de la quimioterapia para el cáncer de mama comprometían el 31,23\% del monto pagado por los EM, y actualmente, con los reajustes en el período, esta pasó a comprometer el 100,75\%. Al proyectar los reajustes para los próximos años, se encontró que el presupuesto estaría comprometido en 121,6\%, tomando en cuenta el mismo escenario inflacionario. Se concluyó que la forma actual de financiar el tratamiento de quimioterapia para el cáncer de mama no resultó ser financieramente sostenible en el escenario evaluado y que si no se realizan nuevas inversiones, las pacientes del SUS no se beneficiarán de las nuevas tecnologías de tratamiento.

Palabras clave: Cáncer; Tratamiento; SUS; Financiación.

\section{Introdução}

O câncer é uma das quatro principais causas de morte no mundo, a segunda entre as doenças e agravos não transmissíveis, constituindo-se no maior problema de saúde pública em função do crescimento de novos casos a cada ano. Segundo a Organização Mundial da Saúde, em 2020 foi estimado um número de 19.292 .789 novos casos de canceres em ambos os sexos por todo mundo, sendo o câncer de mama o mais prevalente entre todos com uma estimativa de 2.261 .419 novos casos (World Health Organization, 2021).

Os cânceres de pulmão e de mama são de maior incidência com aproximadamente 2,1 milhões de novos casos a cada ano. A estimativa para o Brasil é de ocorrer 1.875 mil novos casos no período de 2020 a 2022, perfazendo um incremento de 625 mil por ano. Desses, o de mama, se destaca negativamente com cerca de 10,5\% da previsão, ficando atrás apenas do câncer de pele não melanoma (Bray et al, 2018; Instituto Nacional do Câncer, 2021).

Sabe-se que o diagnóstico precoce do câncer é determinante para o sucesso do tratamento, seja na forma cirúrgica, radioterápica e/ou quimioterápica (Barrios et al, 2021). Ocorre que as unidades públicas credenciadas para atenção oncológica apresentam dificuldades para garantir o acesso rápido ao diagnóstico e tratamento em função da forma de organização e de financiamento das ações. É importante ressaltar que um dos fatores que devem ser levados em consideração no tratamento oncológico é o custo elevado. Em muitos países em desenvolvimento socioeconômico, o valor do tratamento impacta 
diretamente no acesso aos serviços de saúde. Por não ter uma organização e sistematização para os atendimentos oncológicos no serviço público, não há certeza no fornecimento de um tratamento seguro, eficiente e eficaz (Silva et. al., 2019).

Nas últimas décadas no Brasil, a evolução das ações públicas empreendidas para redução da mortalidade e incapacidades avançaram significativamente, em todos os níveis de complexidade, com o surgimento do Sistema Único de Saúde - SUS. De acordo com seus princípios e diretrizes, as ações de promoção, prevenção, detecção precoce, tratamento oportuno e cuidados paliativos devem acontecer por meio de uma rede de atenção especializada (Brasil, 2014).

Tais ações devem ser contratualizadas e financiadas diretamente pelo Ministério da Saúde - MS com o apoio das Secretarias Estaduais e Municipais de Saúde, da Atenção Primária à Especializada, através de uma tabela de procedimentos e custos. Os valores estabelecidos pelo MS para cada procedimento devem ser suficientes para custear todas as despesas para realização dos tratamentos, incluindo insumos (como medicamentos), pessoas envolvidas, instalações prediais e equipamentos (Brasil, 2014).

No caso dos procedimentos quimioterápicos, salvo algumas exceções definidas pelo MS, não se especifica ou limita os medicamentos a serem utilizados nos tratamentos, ficando a responsabilidade de realizar a seleção, aquisição e dispensação para cada Unidade de Saúde, estando ainda livres para definir seus protocolos de tratamento. Além das dificuldades apresentadas, os valores da maioria dos procedimentos encontram-se congelados há anos, não acompanhando os reajustes dos preços dos insumos, salários, e energia, por exemplo. Nesse cenário fica perceptível que há um aumento constante no desequilíbrio entre receita e despesa. Essa situação gera insatisfação dos gestores, que com muitas dificuldades precisam fazer constantemente ajustes para manter os serviços. Isso implica na qualidade do atendimento ao usuário por restringir acesso às novas tecnologias, culminando com frequência na judicialização para acesso a medicamentos, outros produtos e serviços (Vieira, 2020).

Em empresas e organizações, incluindo as da área da saúde, que possuem uma sustentabilidade financeira, há equilíbrio entre receita e despesa de forma a garantir o custeio das suas necessidades presentes e futuras de forma a permanecer no mercado por longo prazo (Amorim, 2009; Fachini, 2005). Nesse caso, a receita deve ser suficiente para garantir o custeio e manter reserva para as emergências, que podem acontecer com frequência nos estabelecimentos de saúde, em consequência das inovações tecnológicas que trazem custos significativos.

Considerando isso, os recursos disponibilizados pelo Ministério da Saúde, aos estabelecimentos credenciados, parecem não ser suficiente para custeio integral do tratamento oncológico por quimioterapia. Nesse cenário, buscou-se analisar a Rede de Atenção em Oncologia do SUS em Pernambuco, diante da atual forma de financiamento e valores de repasse atualmente realizada pelo SUS. Foi também realizado um recorte para verificar o financiamento e o custo do tratamento quimioterápico do câncer de mama em um hospital referência. Com isso, pretendeu-se avaliar se é possível garantir a oferta de serviços de qualidade aos usuários, em acordo com a legislação vigente, e contribuir para ampliar os debates neste importante seguimento da saúde.

\section{Metodologia}

Foi realizado um estudo observacional, analítico e retrospectivo, em que se analisou a produção e os custos dos procedimentos em quimioterapia dos estabelecimentos da Rede de Atenção em Oncologia do Estado de Pernambuco, credenciados no período de 2015 a 2020, disponíveis no sistema TabWin do MS. Dos treze estabelecimentos credenciados, três não dispunham de dados no sistema TabWin e por este motivo apenas dez estabelecimentos foram incluídos neste estudo.

Com o intuito de sistematizar os objetivos propostos, foi realizada busca nas bases da Biblioteca Virtual em Saúde que inclui SciELO e Lilacs; e Medline/PubMed, utilizando os descritores: "Câncer", "Financiamento", "Etiologia", "SUS", "Diagnóstico" e "Tratamento" de forma individual ou as permutando e as combinando entre si, nos campos título, resumo ou 
assunto; incluindo artigos originais, teses e dissertações em português. Fontes complementares incluíram as referências dos artigos selecionados e indicações de conhecimento do próprio autor (Estrela, 2018).

As informações a respeito da transferência de recursos federais diretamente aos municípios ou estados, e às relacionadas aos esquemas terapêuticos e valores repassados, foram obtidas no aplicativo TABNET (http://tabnet.saude.pe.gov.br/), desenvolvido pelo DATASUS, que é um tabulador de domínio público que permite formular consultas às bases de dados do Sistema Único de Saúde. As informações sobre a incorporação de medicamentos para câncer de mama foram avaliadas no site da Comissão Nacional de Incorporação de Tecnologias - CONITEC (http://conitec.gov.br/home).

Para a análise de dados se considerou os valores repassados para os tratamentos dos procedimentos quimioterápicos utilizados em cada instituição. Foi utilizada a ferramenta Excel, pertencente ao pacote Office 2010, para analisar e comparar a evolução dos valores repassados para os procedimentos quimioterápicos.

\section{Resultados}

Foi verificado que, no período entre os anos de 2015 e 2020, realizaram-se 321.286 procedimentos quimioterápicos, relacionados a diferentes tipos de cânceres, nos estabelecimentos que compõem a Rede de Atenção Oncológica do Estado de Pernambuco. Na Tabela 1 observa-se a evolução quantitativa de procedimentos quimioterápicos realizados pelos estabelecimentos de saúde de Pernambuco entre os anos de 2015 e 2020.

Tabela 1: Evolução quantitativa de procedimentos quimioterápicos realizados pelos estabelecimentos de saúde de Pernambuco entre os anos de 2015 e 2020.

\begin{tabular}{cccccccccc}
\hline & 2015 & 2016 & 2017 & 2018 & 2019 & 2020 & TOTAL & \% \\
\hline HCP & 12.158 & 13.337 & 14.335 & 15.467 & 15.913 & 16.214 & 87.424 & $27,21 \%$ \\
IMIP & 12.567 & 12.049 & 11.745 & 9.983 & 10.552 & 9.590 & 66.486 & $20,69 \%$ \\
HUOC & 6.791 & 6.803 & 6.945 & 7.410 & 8.382 & 9.154 & 45.485 & $14,16 \%$ \\
HEMOPE & 6.352 & 7.205 & 7.734 & 7.712 & 7.943 & 7.904 & 44.850 & $13,96 \%$ \\
HC/UFPE & 2.977 & 3.486 & 2.609 & 3.781 & 3.989 & 4.181 & 21.023 & $6,54 \%$ & $6,51 \%$ \\
HRA & 3.377 & 3.161 & 3.258 & 3.622 & 3.648 & 3.851 & 20.917 & 15.672 & $4,88 \%$ \\
HDM & 3.481 & 4.363 & 4.304 & 3.524 & - & - & 2.234 & 11.519 & $3,59 \%$ \\
HBL & 1.581 & 1.818 & 2.111 & 1.735 & 2.040 & 1.177 & 4.194 & $1,31 \%$ \\
HMA & - & 119 & 664 & 1.011 & 1.223 & 1.19 & 3.716 & $1,16 \%$ \\
NSPS & 552 & 685 & 669 & 731 & 604 & 475 & 54.286 & - \\
TOTAL & 49.836 & 53.026 & 54.374 & 54.976 & 54.294 & 54.780 & 321.286 \\
\hline
\end{tabular}

HCP: Hospital do Câncer de Pernambuco; IMIP: Instituto Materno Infantil de Pernambuco; HUOC: Hospital Universitário Oswaldo Cruz; HEMOPE: Fundação de Hemoterapia de Pernambuco; HC/UFPE: Hospital das Clínicas; HRA: Hospital Regional do Agreste; HBL: Hospital Barão de Lucena; Hospital Memorial Arcoverde - HMA; NSPS: Casa de Saúde Nossa Senhora do Perpetuo Socorro; Fonte: Elaborado pelos autores baseado nos dados extraídos através da plataforma TABNET.

Dentre os estabelecimentos avaliados, o HCP foi o que mais realizou quimioterapias no período, totalizando em 87.424 procedimentos do total de 321.286, o que representou 27,25\% de todos os procedimentos realizados em Pernambuco. O HCP também teve uma maior média crescente evolutiva de procedimentos realizados entre todos os estabelecimentos ao longo dos anos $(0,05 \%)$. Já o IMIP apresentou a segunda maior prevalência (20,69\%), seguido do HUOC (14,16\%), do HEMOPE $(13,96 \%)$ e do HC-UFPE $(6,54 \%)$, como observado na tabela acima. 
A Figura 1 apresenta Monetização dos procedimentos quimioterápicos recebidos pelos estabelecimentos da Rede de Atenção Oncológica de Pernambuco, entre os anos de 2015 e 2020.

Figura 1: Monetização dos procedimentos quimioterápicos recebidos pelos estabelecimentos da Rede de Atenção Oncológica de Pernambuco, entre os anos de 2015 e 2020.

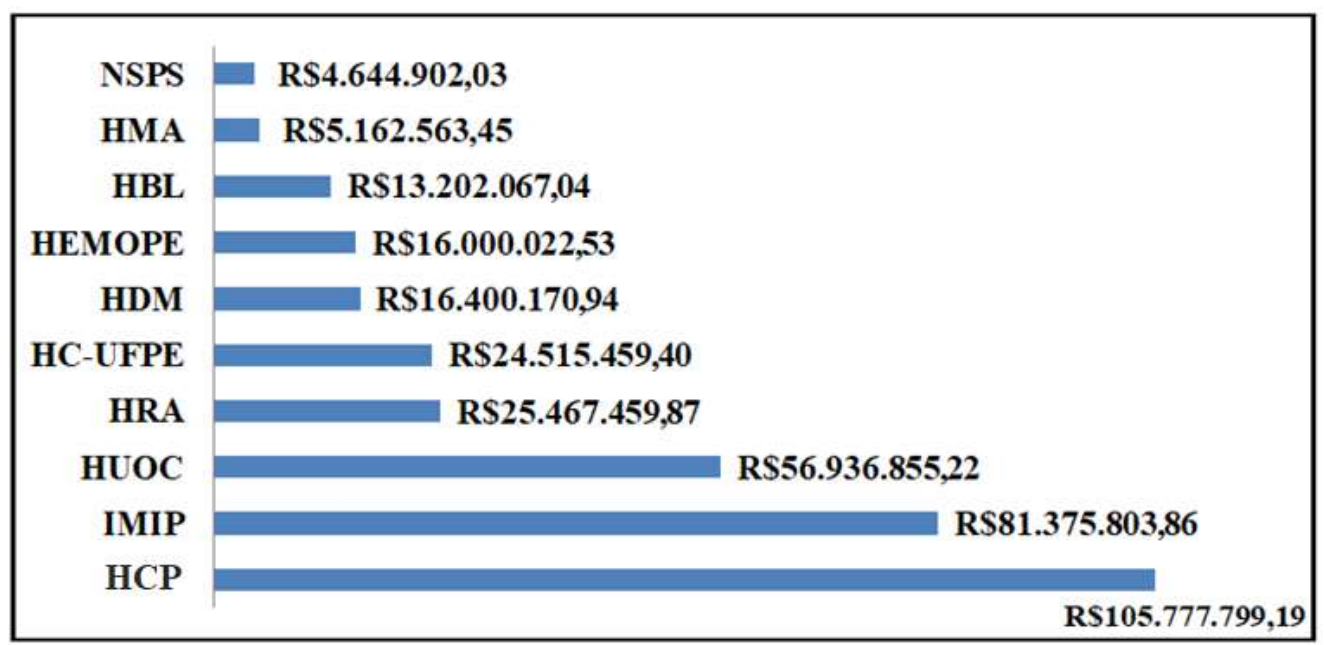

Fonte: Elaborado pelos autores baseado nos dados extraídos através da plataforma TABNET.

Referente à monetização destes procedimentos, o HCP continua possuindo maior predomínio quantitativo, acumulando o total de $\mathrm{R} \$ 105.777 .799,19$ (30,27\%), sendo acompanhado pelo IMIP (R \$81.375.803,86; 23,28\%), HUOC (R \$56.936.855,22; 16,29\%), HRA (R $\$ 25.467 .459,87 ; 7,29 \%)$ e pelo HC/UFPE (R $\$ 24.515 .459,40 ; 7,01 \%)$.

Com relação aos custos dos procedimentos quimioterápicos, Figura 2, foi identificado que 31,54\% das despesas da Rede de Atenção em Oncologia do Estado de Pernambuco destinadas às quimioterapias (R\$ 381.675.517,70) foram despendidas com o tratamento do câncer de mama, sendo elencada como a neoplasia mais onerosa para o Estado, no período avaliado. Na Figura 2 é possível observar o custo das 10 neoplasias mais caras dentro da Rede avaliada. 
Research, Society and Development, v. 10, n. 16, e459101623883, 2021

(CC BY 4.0) | ISSN 2525-3409 | DOI: http://dx.doi.org/10.33448/rsd-v10i16.23883

Figura 2: Ranking das neoplasias mais onerosas para Rede de Atenção em Oncologia do Estado de Pernambuco, realizados no período de 2015 a 2020.

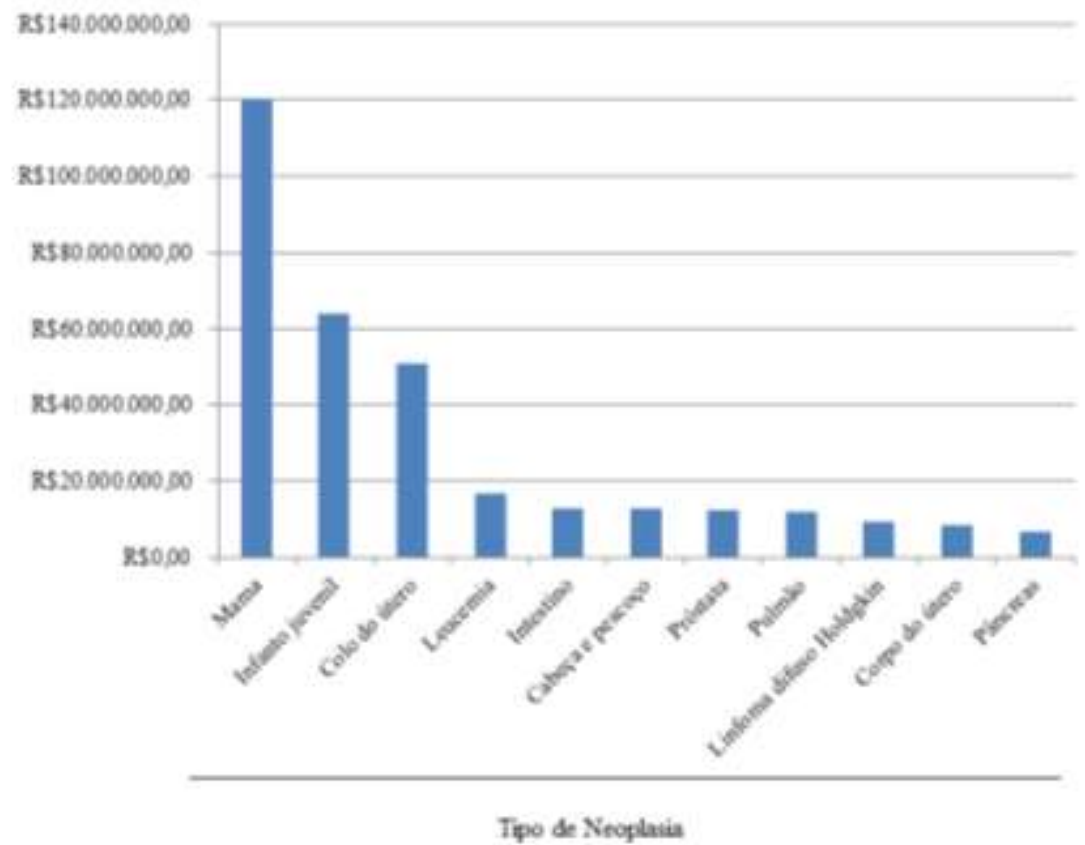

Fonte: Elaborado pelos autores baseado nos dados extraídos através da plataforma TABNET.

Sabendo que o câncer de mama foi a neoplasia mais onerosa, seguida de tumores pediátricos e colo do útero, foram analisados os custos dos procedimentos quimioterápicos para essa enfermidade. Foram identificados 13 procedimentos relacionados ao câncer de mama, como mostra a Tabela 2. 
Tabela 2: Relação de procedimentos quimioterápicos para o câncer de mama, realizados no período de 2015 a 2020 no Estado de Pernambuco.

\begin{tabular}{|c|c|c|}
\hline PROCEDIMENTOS QUIMIOTERÁPICOS & $\begin{array}{c}\text { NÚMERO DE } \\
\text { PROCEDIMENTOS } \\
\text { REALIZADOS } \\
\end{array}$ & $\%$ \\
\hline QUIMIOTERAPIA DO CARCINOMA DE MAMA (PRÉVIA) & 25413 & $27,93 \%$ \\
\hline QUIMIOTERAPIA DO CARCINOMA DE MAMA AVANÇADO - $2^{\mathrm{a}}$ LINHA & 13999 & $15,39 \%$ \\
\hline QUIMIOTERAPIA DO CARCINOMA DE MAMA AVANÇADO - $1^{\mathrm{a}}$ LINHA & 11953 & $13,14 \%$ \\
\hline QUIMIOTERAPIA DO CARCINOMA DE MAMA EM ESTÁDIO II & 11864 & $13,04 \%$ \\
\hline QUIMIOTERAPIA DO CARCINOMA DE MAMA EM ESTÁDIO III & 6985 & $7,68 \%$ \\
\hline $\begin{array}{l}\text { MONOQUIMIOTERAPIA DO CARCINOMA DE MAMA HER-2 POSITIVO EM ESTÁDIO III } \\
\text { (ADJUVANTE) }\end{array}$ & 6237 & $6,86 \%$ \\
\hline $\begin{array}{l}\text { MONOQUIMIOTERAPIA DO CARCINOMA DE MAMA HER-2 POSITIVO EM ESTÁDIO II } \\
\text { (ADJUVANTE) }\end{array}$ & 3823 & $4,20 \%$ \\
\hline $\begin{array}{l}\text { POLIQUIMIOTERAPIA DO CARCINOMA DE MAMA HER-2 POSITIVO EM ESTÁDIO III } \\
\text { (PRÉVIA) }\end{array}$ & 3351 & $3,68 \%$ \\
\hline QUIMIOTERAPIA DO CARCINOMA DE MAMA EM ESTÁDIO I & 3008 & $3,31 \%$ \\
\hline $\begin{array}{l}\text { POLIQUIMIOTERAPIA DO CARCINOMA DE MAMA HER-2 POSITIVO EM ESTÁDIO II } \\
\text { (ADJUVANTE) }\end{array}$ & 1521 & $1,67 \%$ \\
\hline $\begin{array}{l}\text { POLIQUIMIOTERAPIA DO CARCINOMA DE MAMA HER-2 POSITIVO EM ESTÁDIO III } \\
\text { (ADJUVANTE) }\end{array}$ & 1310 & $1,44 \%$ \\
\hline $\begin{array}{l}\text { MONOQUIMIOTERAPIA DO CARCINOMA DE MAMA HER-2 POSITIVO EM ESTÁDIO I } \\
\text { (ADJUVANTE) }\end{array}$ & 839 & $0,92 \%$ \\
\hline $\begin{array}{l}\text { POLIQUIMIOTERAPIA DO CARCINOMA DE MAMA HER-2 POSITIVO EM ESTÁDIO I } \\
\text { (ADJUVANTE) }\end{array}$ & 669 & $0,74 \%$ \\
\hline
\end{tabular}

Fonte: Elaborado pelos autores baseado nos dados extraídos através da plataforma TABNET.

No período apontado, um total de 90.972 tratamentos quimioterápicos para o câncer de mama foi realizado pela Rede de Atenção Oncológica de Pernambuco, com exceção do HEMOPE, que é um estabelecimento especializado em tratamento de onco-hematologia. Observa-se um maior predomínio para o tratamento intitulado "QUIMIOTERAPIA DO CARCINOMA DE MAMA (PRÉVIA)", no qual inclui medicamentos como adriamicina (doxorrubicina), ciclofosfamida e paclitaxel, que representa $27,93 \%$ dos procedimentos realizados.

A Tabela 3 traz a relação de procedimentos quimioterápicos utilizados para o câncer de mama e a respectiva monetização e impacto ao longo dos anos (5 anos). 
Tabela 3: Relação de procedimentos quimioterápicos utilizados para o câncer de mama e a respectiva monetização e impacto ao longo dos anos (5 anos).

\begin{tabular}{|c|c|c|c|}
\hline PROCEDIMENTOS QUIMIOTERÁPICOS & $\begin{array}{l}\text { VALORES } \\
\text { INDIVIDUAL } \\
(\mathrm{R} \$) \\
\end{array}$ & $\begin{array}{c}\text { VALORES } \\
\text { REPASSADOS } \\
(\mathrm{R} \$) \\
\end{array}$ & $\%$ \\
\hline QUIMIOTERAPIA DO CARCINOMA DE MAMA (PRÉVIA) & $1.400,00$ & $35.578 .200,00$ & 31,29 \\
\hline QUIMIOTERAPIA DO CARCINOMA DE MAMA AVANÇADO - $2^{\mathrm{a}}$ LINHA & $2.378,90$ & $33.302 .221,10$ & 29,29 \\
\hline QUIMIOTERAPIA DO CARCINOMA DE MAMA AVANÇADO $-1^{\mathrm{a}}$ LINHA & $1.700,00$ & $20.320 .100,00$ & 17,87 \\
\hline QUIMIOTERAPIA DO CARCINOMA DE MAMA EM ESTÁDIO II & 800,00 & $9.491 .200,00$ & 8,35 \\
\hline QUIMIOTERAPIA DO CARCINOMA DE MAMA EM ESTÁDIO III & 800,00 & $5.588 .000,00$ & 4,91 \\
\hline $\begin{array}{l}\text { POLIQUIMIOTERAPIA DO CARCINOMA DE MAMA HER-2 POSITIVO } \\
\text { EM ESTÁDIO III (PRÉVIA) }\end{array}$ & $1.400,00$ & $4.691 .400,00$ & 4,13 \\
\hline QUIMIOTERAPIA DO CARCINOMA DE MAMA EM ESTÁDIO I & 571,50 & 1.719.072,00 & 1,51 \\
\hline $\begin{array}{l}\text { POLIQUIMIOTERAPIA DO CARCINOMA DE MAMA HER-2 POSITIVO } \\
\text { EM ESTÁDIO II (ADJUVANTE) }\end{array}$ & 800,00 & $1.216 .800,00$ & 1,07 \\
\hline $\begin{array}{l}\text { POLIQUIMIOTERAPIA DO CARCINOMA DE MAMA HER-2 POSITIVO } \\
\text { EM ESTÁDIO III (ADJUVANTE) }\end{array}$ & 800,00 & $1.048 .000,00$ & 0,92 \\
\hline $\begin{array}{l}\text { POLIQUIMIOTERAPIA DO CARCINOMA DE MAMA HER-2 POSITIVO } \\
\text { EM ESTÁDIO I (ADJUVANTE) }\end{array}$ & 571,50 & $382.333,50$ & 0,34 \\
\hline $\begin{array}{l}\text { MONOQUIMIOTERAPIA DO CARCINOMA DE MAMA HER-2 POSITIVO } \\
\text { EM ESTÁDIO III (ADJUVANTE) }\end{array}$ & 34,10 & $212.681,70$ & 0,19 \\
\hline $\begin{array}{l}\text { MONOQUIMIOTERAPIA DO CARCINOMA DE MAMA HER-2 POSITIVO } \\
\text { EM ESTÁDIO II (ADJUVANTE) }\end{array}$ & 34,10 & $130.364,30$ & 0,11 \\
\hline $\begin{array}{l}\text { MONOQUIMIOTERAPIA DO CARCINOMA DE MAMA HER-2 POSITIVO } \\
\text { EM ESTÁDIO I (ADJUVANTE) }\end{array}$ & 34,10 & $28.609,90$ & 0,03 \\
\hline
\end{tabular}

Fonte: Elaborado pelos autores baseado nos dados extraídos através da plataforma TABNET.

Para verificar se os recursos disponibilizados pelo Ministério da Saúde são suficientes para custeio integral do tratamento oncológico por quimioterapia, foi realizada uma análise em um hospital referência pertencente à Rede de Atenção Oncológica de Pernambuco. Ao levantar os valores pagos por procedimento entre os anos selecionados, é possível observar que estes se mantêm, variando apenas o montante financeiro de acordo com a quantidade de procedimentos atendidos pelas instituições de saúde, não havendo uma evolução financeira de acordo com o mercado ou com a inflação para a aquisição dos medicamentos ou matérias que englobam determinado procedimento.

Atualmente estão vigentes 21 procedimentos para o tratamento do câncer de mama em adultos no SUS. Destes, 6 são utilizados na quimioterapia paliativa, 3 procedimentos para quimioterapia prévia (neoadjuvante / citorredutora) e 12 para quimioterapia adjuvante (profilática). Na Figura 3 se observa que os valores pagos para custear os medicamentos quimioterápicos, independentemente do tipo, não têm sofrido reajustes ao longo dos anos, de acordo com a tabela do SUS. 
Research, Society and Development, v. 10, n. 16, e459101623883, 2021

(CC BY 4.0) | ISSN 2525-3409 | DOI: http://dx.doi.org/10.33448/rsd-v10i16.23883

Figura 3: Custos despendidos com quimioterápicos para adultos com câncer de mama, de acordo com a tabela do SUS.

a. Quimioterapia Paliativa

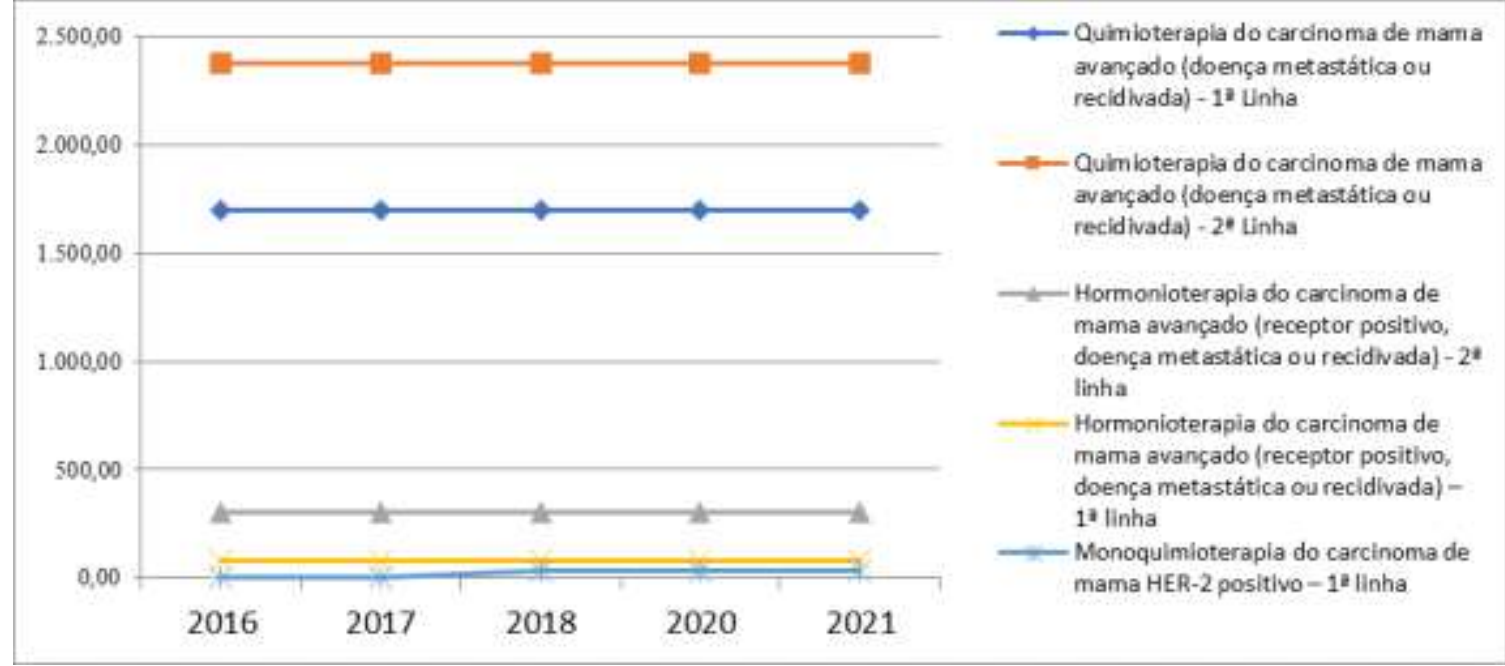

b. Quimioterapia Prévia (Neoadjuvante/Citorredutora)

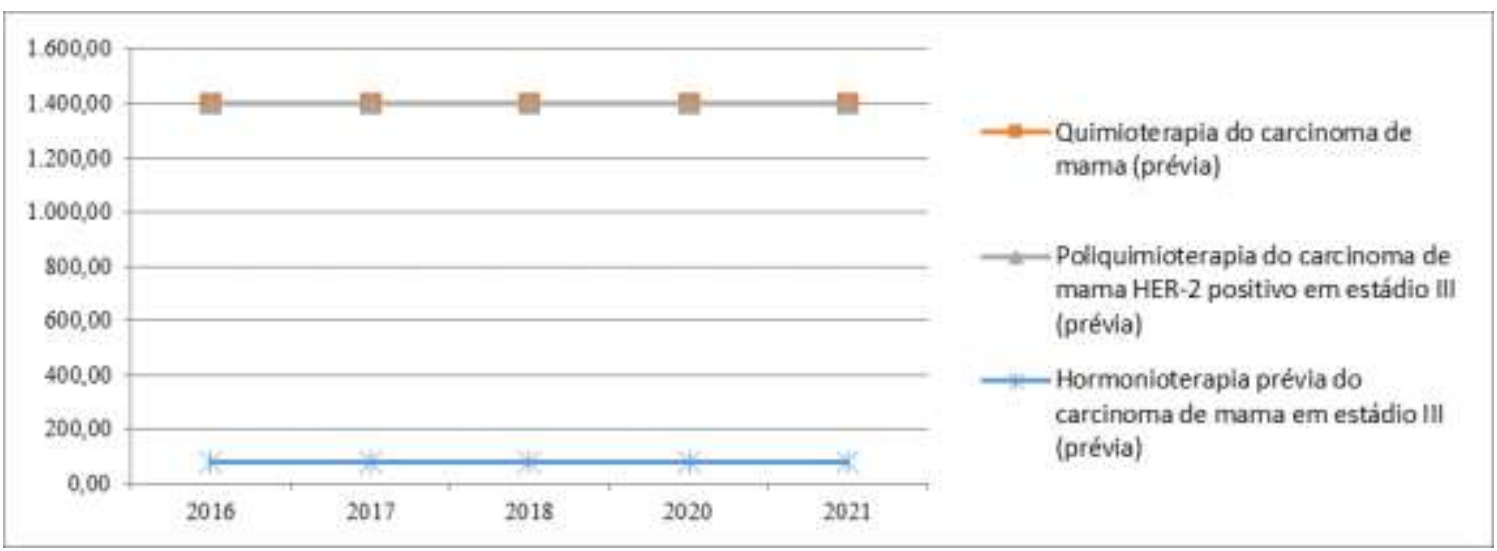

c. Quimioterapia Adjuvante (Profilática)

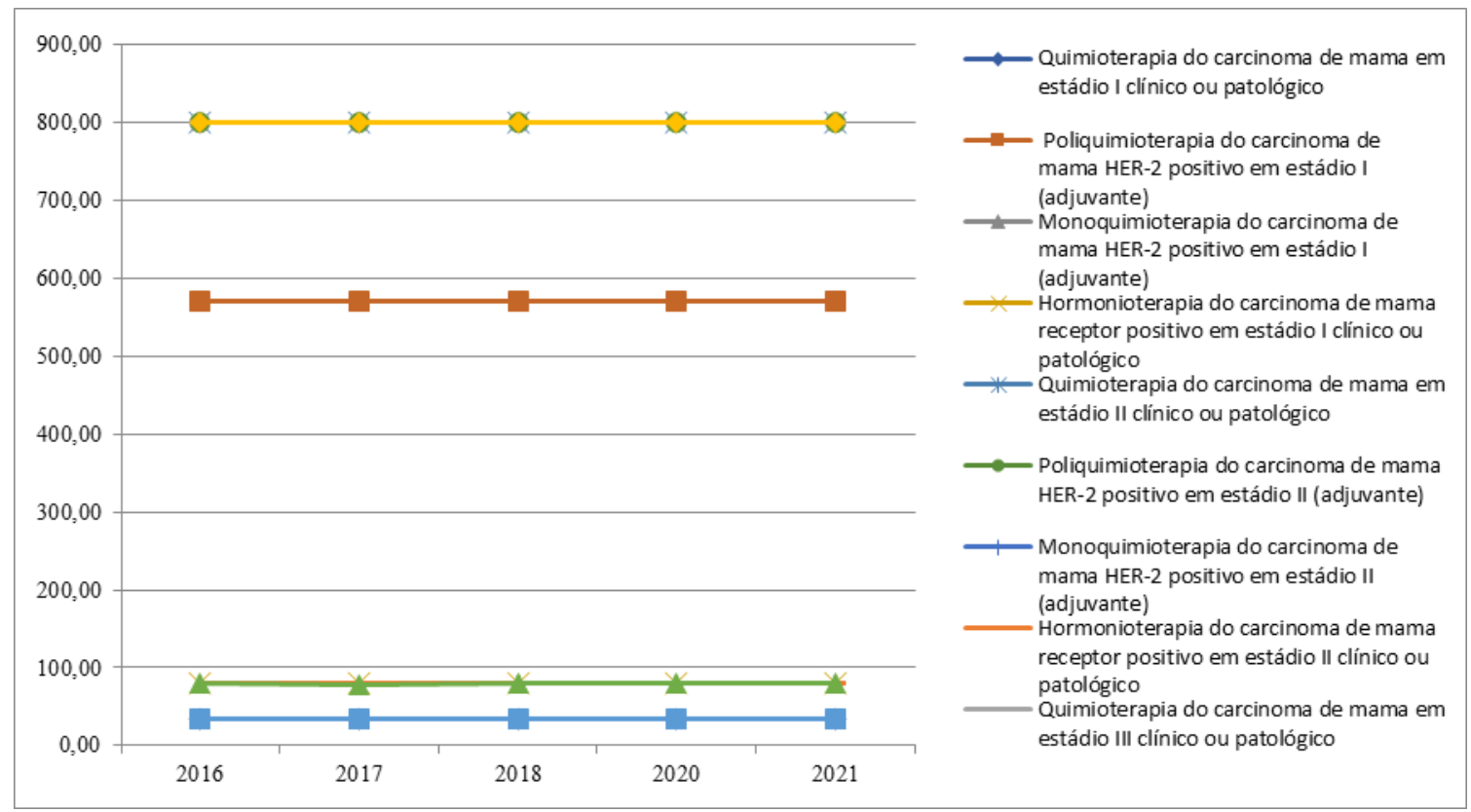

Fonte: Autores (2021). 
Para corroborar com este achado, a Tabela 4 traz os custos dos esquemas quimioterápicos adjuvantes e neoadjuvante para o câncer de mama em diferentes períodos. Ao comparar os custos desses esquemas entre os períodos de 2011 e 2020 , observou-se que os mesmos tiveram um aumento significativo. Os custos com os esquemas terapêuticos AC-T, AC-D, FAC, FEC e AC-TH, aumentaram 215,96\%, 221,63\%, 218,98\%, 259,80\% e 315,96\%, respectivamente, no período (2011 a 2020). Em 2011 apenas os custos dos medicamentos quimioterápicos comprometiam 31,23\% do valor pago pelo MS do tratamento completo. Atualmente, com os reajustes do período, passaram a comprometer 100,75\%,, no esquema AC-T, por exemplo. Já com os esquemas AC-D, FAC, FEC e AC-TH, houve comprometimento de 32,6\%, 31,58\%, 65,33\% e 54,89\% dos valores pagos pelo MS, respectivamente, com os custos dos medicamentos.

Tabela 4: Custos relacionados aos esquemas quimioterápicos adjuvantes e neoadjuvante para o câncer de mama, durante os anos de 2011 e 2020, e receita prevista financiada pelo MS.

\begin{tabular}{|c|c|c|c|c|c|c|c|}
\hline \multirow{2}{*}{\multicolumn{2}{|c|}{$\begin{array}{c}\text { ES QUEMAS } \\
\text { ESQ UEMAS ADJUVANTES }\end{array}$}} & \multirow{2}{*}{\multicolumn{2}{|c|}{ CUSTO POR CICLO }} & \multicolumn{3}{|c|}{ PROCEDIMENTO } & \multirow{2}{*}{$\begin{array}{l}\text { RECEITA } \\
\text { PREVIS TA }\end{array}$} \\
\hline & & & & Código & & Valor & \\
\hline \multicolumn{2}{|c|}{ AC-T } & 2011 & 2020 & \multirow{6}{*}{$03.04 .04 .028-8$} & \multirow{6}{*}{ RS } & \multirow{6}{*}{800,00} & \multirow{6}{*}{ R\$ $4.800,00$} \\
\hline Adriamicina: $60 \mathrm{mg} / \mathrm{m} 2$ & D1 & R\$ 36,40 & RS 90,00 & & & & \\
\hline Ciclofosfamida: $600 \mathrm{mg} / \mathrm{m} 2$ & D1 a cada 21 dias $\times 4$ ciclos & RS 22,98 & RS 43,36 & & & & \\
\hline \multirow[t]{3}{*}{ Paclitaxel: $80 \mathrm{mg} / \mathrm{m} 2$} & D1 após 4 ciclos de $\mathrm{AC}$ & 36,57 & 71,37 & & & & \\
\hline & Custo por ciclo & RS 95,95 & RS 204,73 & & & & \\
\hline & Custo tratamento & RS $1.151,40$ & RS $2.456,76$ & & & & \\
\hline \multicolumn{2}{|c|}{ AC-D } & 2011 & 2020 & \multirow{6}{*}{$03.04 .04 .028-8$} & \multirow{6}{*}{ RS } & \multirow{6}{*}{800,00} & \multirow{6}{*}{ R\$ $4.800,00$} \\
\hline Adriamicina: $60 \mathrm{mg} / \mathrm{m} 2$ & D1 & R\$ 36,40 & RS 90,00 & & & & \\
\hline Ciclofosfamida: $600 \mathrm{mg} / \mathrm{m} 2$ & D1 a cada 21 dias $\times 4$ ciclos & 22,98 & RS 43,36 & & & & \\
\hline \multirow[t]{3}{*}{ Docetaxel: $100 \mathrm{mg} / \mathrm{m} 2$} & D1 após 4 ciclos de $\mathrm{AC}$ & R\$ 118,00 & RS 258,00 & & & & \\
\hline & Custo por ciclo & RS 177,38 & RS 391,36 & & & & \\
\hline & Custo tratamento & RS 709.52 & RS 1.565 .44 & & & & \\
\hline \multicolumn{2}{|c|}{ FAC } & 2011 & 2020 & \multirow{6}{*}{$03.04 .04 .028-8$} & \multirow{6}{*}{ RS } & \multirow{6}{*}{800,00} & \multirow{6}{*}{ R\$ $3.200,00$} \\
\hline 5-Fluorouracil: $500 \mathrm{mg} / \mathrm{m} 2$ & D1 & R\$ 17,54 & RS $\quad 35,08$ & & & & \\
\hline Adriamicina: $60 \mathrm{mg} / \mathrm{m} 2$ & D1 & 36,40 & RS 90,00 & & & & \\
\hline \multirow[t]{3}{*}{ Ciclofosfamida: $600 \mathrm{mg} / \mathrm{m} 2$} & D1 a cada 21 dias $\times 6$ ciclos & 22,98 & RS 43,36 & & & & \\
\hline & Custo por ciclo & 76,92 & RS 168,44 & & & & \\
\hline & Custo tratamento & RS 461,52 & RS $1.010,64$ & & & & \\
\hline & $\mathrm{CC}$ & 2011 & 2020 & & & & \\
\hline 5-Fluorouracil: $500 \mathrm{mg} / \mathrm{m} 2$ & D1 & R\$ 17,54 & RS $\quad 35,08$ & & & & \\
\hline Epirrubicina: $100 \mathrm{mg} / \mathrm{m} 2$ & D1 & 93,60 & RS 260,00 & $03.0404028-8$ & RS & 800,00 & R\$ 3.20000 \\
\hline Ciclofosfamida: $600 \mathrm{mg} / \mathrm{m} 2$ & D1 a cada 21 dias $\times 6$ ciclos & 22,98 & RS 43,36 & & & & \\
\hline & Custo por ciclo & RS 134,12 & RS $\quad 338,44$ & & & & \\
\hline & Custo tratamento & RS 804,72 & RS $2.090,64$ & & & & \\
\hline ESQ UEMA NE & OADJUVANTE & & & & & & \\
\hline $\mathbf{A C}$ & -TH & 2011 & 2020 & & & & \\
\hline Adriamicina: $60 \mathrm{mg} / \mathrm{m} 2$ & D1 & 36,40 & RS $\quad 90,00$ & $030404018-5$ & $B S$ & 1.40000 & BS 840000 \\
\hline Ciclofosfamida: $600 \mathrm{mg} / \mathrm{m} 2$ & D1 a cada 21 dias $\times 4$ ciclos & 22,98 & 43,36 & $03.04 .04 .010-5$ & $\mathrm{Ko}$ & $1.400,00$ & Ko $0.400,00$ \\
\hline Paclitaxel: 80mg/m2 & $D 1$ após 4 ciclos de $A C$ & R\$ 36,57 & RS 71,37 & & & & \\
\hline $\begin{array}{l}\text { Trastuzumabe: } 4 \mathrm{mg} / \mathrm{kg} \\
\text { Trastuzumabe: } 2 \mathrm{mg} / \mathrm{kg}\end{array}$ & $\begin{array}{l}\text { Ataque } \\
\text { Semanal por } 12 \text { meses }\end{array}$ & Repassado & to MS sem & $03.04 .05 .029-6$ & RS & 34,11 & RS 409,32 \\
\hline & Custo por ciclo & $\mathrm{R} \$ \quad 95,95$ & RS 204,73 & & & & \\
\hline & Custo tratamento & RS 1.151 .40 & RS $2.456,76$ & & & Total & RS 8.809 .32 \\
\hline
\end{tabular}

Fonte: Elaborado pelos autores baseado nos dados extraídos através da plataforma TABNET. 
Foi realizada uma projeção baseada no reajuste do preço dos quimioterápicos, utilizados nos procedimentos quimioterápicos do tipo adjuvante e neoadjuvante, adquiridos por um hospital referência no período entre 2011 e 2020 . A partir daí, assumiu-se essa variação, considerada como reajuste, para projetar os preços futuros para os anos de 2030 e 2040 , como mostra a Figura 4.

Figura 4: Projeção do custo de procedimentos quimioterápicos Adjuvantes (A) e Neoadjuvantes (B) em um hospital referência.
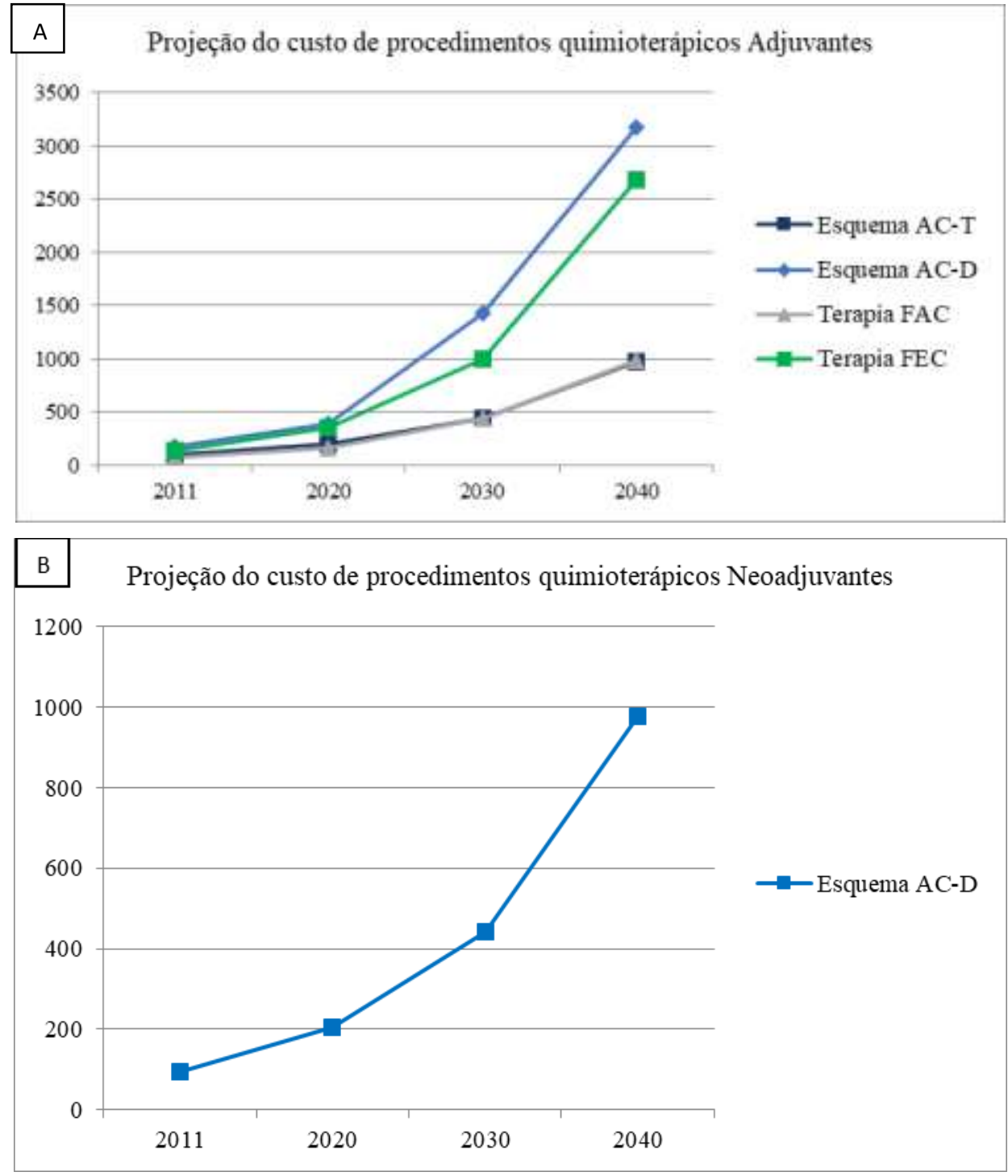

Fonte: Autores (2021).

Ao observar a Figura 4-A, é possível verificar que os custos com cada esquema adjuvante apresentado aumentariam consideravelmente se houvesse o mesmo reajuste nos anos subsequentes. Para exemplificar, verificou-se que o custo com a terapia FAC teve reajuste monetário em 118,98\%, entre os anos de 2011 e 2020, ao projetar esse reajuste, levando em conta o mesmo cenário inflacionário, observou-se que os custos com essa mesma terapia aumentariam em 121,6\% entre o período de 2030 a 2040. Para os demais esquemas e adjuvantes o cenário é ainda pior. Na Figura 4-B, observa-se a projeção dos custos com o Esquema Neoadjuvante AC-D. Nesse caso, entre 2011 e 2020 o custo desse esquema teve 113,4\% de reajuste. Levando 
em conta o mesmo cenário inflacionário e o mesmo reajuste, observou-se que os custos com esse esquema aumentariam em $120,1 \%$ no período entre 2030 a 2040.

Além disso, foi realizado um levantamento para verificar a incorporação de tecnologias pela CONITEC, no que diz respeito à quimioterapia direcionada para o câncer de mama, como mostra a Tabela 5.

Tabela 5: Recomendações sobre a incorporação de tecnologias para a terapia do câncer de mama no SUS.

\begin{tabular}{|c|c|c|c|c|c|c|c|}
\hline TECNOLOGIA & $\begin{array}{l}\text { INDICAÇÃO } \\
\text { TERAFÊUTICA }\end{array}$ & $\begin{array}{c}\text { DECISẪO DA } \\
\text { CONITEC }\end{array}$ & $\begin{array}{c}\text { TIPO DE } \\
\text { TECNOLOGIA }\end{array}$ & DEMANDANTE & $\begin{array}{l}\text { CONSULTA } \\
\text { PÚBLICA }\end{array}$ & $\begin{array}{c}\text { CATEGORIA DE } \\
\text { DOENÇAS (CID- } \\
10) \\
\end{array}$ & $\begin{array}{c}\text { ANO DA } \\
\text { RECOMBNDAÇẨ } \\
0 \\
\end{array}$ \\
\hline Trastuzumabe & $\begin{array}{c}\text { Câncer de mama } \\
\text { avançado }\end{array}$ & Incorporar & Medicamento & $\begin{array}{c}\text { Demanda interna } \\
\text { (Secretaria de } \\
\text { Atençẫo à } \\
\text { Saúde/MS) }\end{array}$ & Sim & $\begin{array}{c}\text { Neoplasias } \\
\text { [tumores] (C00- } \\
\text { D48) }\end{array}$ & 2012 \\
\hline Trastuzumabe & $\begin{array}{c}\text { Câncer de mama } \\
\text { inicial }\end{array}$ & Incorporar & Medicamento & $\begin{array}{c}\text { Demanda interna e } \\
\text { externa (Secretaria } \\
\text { de Atençấo à } \\
\text { SaúdeMS e } \\
\text { Produtos Roche } \\
\text { Químic os e } \\
\text { Farmacêutic os } \\
\text { S.A.) } \\
\end{array}$ & Sim & $\begin{array}{c}\text { Neoplasias } \\
\text { [tumores] (C00- } \\
\text { D48) }\end{array}$ & 2012 \\
\hline Everolimo & $\begin{array}{c}\text { Câncer de mama } \\
\text { avançado na pós- } \\
\text { menopausa }\end{array}$ & Não incorporar & Medicamento & $\begin{array}{c}\text { Demanda externa } \\
\text { (Novartis } \\
\text { Biociências SA.) }\end{array}$ & Sim & $\begin{array}{c}\text { Neoplasias } \\
\text { [tumores] (C00- } \\
\text { D48) }\end{array}$ & 2014 \\
\hline $\begin{array}{c}\text { Hormonioterapia } \\
\text { prévia }\end{array}$ & Câncer de mama & Não incorporar & Procedimento & $\begin{array}{c}\text { Demanda interna } \\
\text { (Secretaria de } \\
\text { Atençẫo à } \\
\text { Saúde/MS) }\end{array}$ & Năo & $\begin{array}{c}\text { Neoplasias } \\
\text { [tumores] (COD- } \\
\text { D48) }\end{array}$ & 2014 \\
\hline \begin{tabular}{c|} 
Diretrizes \\
Nacionais para a \\
Dete cçẫo Precoce \\
do Câncer de Mama
\end{tabular} & Câncer de Mama & Incorporar & PCDT & $\begin{array}{c}\text { Demanda interna } \\
\text { (INCA e Secretaria } \\
\text { de Atençẫo à } \\
\text { Saúde) } \\
\end{array}$ & Sim & $\begin{array}{c}\text { Neoplasias } \\
\text { [tumores] (C00- } \\
\text { D48) }\end{array}$ & 2015 \\
\hline Mamografia & $\begin{array}{l}\text { Rastreamento do } \\
\text { câncer de mama }\end{array}$ & Não incorporar & Procedimento & $\begin{array}{l}\text { Demanda interna } \\
\text { (Secretaria de } \\
\text { Ciência, Tecnologia } \\
\text { e Insumos } \\
\text { Estratégicos/MS) }\end{array}$ & Sim & $\begin{array}{c}\text { Neoplasias } \\
\text { [tumores] (C00- } \\
\text { D48) }\end{array}$ & 2015 \\
\hline $\begin{array}{l}\text { Radioterapia } \\
\text { Intra operatória }\end{array}$ & Tumores de Mama & Não incorporar & Produto & $\begin{array}{c}\text { Demanda externa } \\
\text { (Carl Zeiss do Brasil } \\
\text { Ltda.) }\end{array}$ & Sim & $\begin{array}{c}\text { Neoplasias } \\
\text { [tumores] (C00- } \\
\text { D48) }\end{array}$ & 2016 \\
\hline Pertuzumabe & $\begin{array}{l}\text { Câncer de mama } \\
\text { HER2-positivo } \\
\text { metastático em } \\
\text { primeira linha de } \\
\text { tratamento } \\
\text { associado ao } \\
\text { trastuzumabe e } \\
\text { docetaxel } \\
\end{array}$ & Incorporar & Medicamento & $\begin{array}{c}\text { Demand a externa } \\
\text { (Roche e Sociedade } \\
\text { Brasileira de } \\
\text { Oncologia Clínica) }\end{array}$ & Sim & $\begin{array}{c}\text { Neoplasias } \\
\text { [tumores] (C00- } \\
\text { D48) }\end{array}$ & 2017 \\
\hline Trastuzumabe & $\begin{array}{l}\text { Câncer de mama } \\
\text { HER2-positivo } \\
\text { metastático em } \\
\text { primeira linha de } \\
\text { tratamento }\end{array}$ & Incorporar & Medicamento & $\begin{array}{l}\text { Demanda interna } \\
\text { (Secretaria de } \\
\text { Ciência, Tecnologia } \\
\text { e Insumos } \\
\text { Estratégicos/MS) }\end{array}$ & Sim & $\begin{array}{c}\text { Neoplasias } \\
\text { [tumores] (C00- } \\
\text { D48) }\end{array}$ & 2017 \\
\hline $\begin{array}{c}\text { Diretrizes } \\
\text { Diagnóstica e } \\
\text { Terapêuticas do } \\
\text { Carcinoma de Mama }\end{array}$ & $\begin{array}{c}\text { Carcinoma de } \\
\text { Mama }\end{array}$ & Incorporar & PCDT & $\begin{array}{l}\text { Demanda interna } \\
\text { (Secretaria de } \\
\text { Ciência, Tecnologia } \\
\text { e Insumos } \\
\text { Estratégicos/MS) }\end{array}$ & Sim & $\begin{array}{c}\text { Neoplasias } \\
\text { [tumores] (C00- } \\
\text { D48) }\end{array}$ & 2018 \\
\hline $\begin{array}{c}\text { Diretrizes } \\
\text { Diagnóstic a e } \\
\text { Terapêuticas do } \\
\text { Carcinoma de Mama }\end{array}$ & $\begin{array}{c}\text { Carcinoma de } \\
\text { Mama }\end{array}$ & Incorporar & PCDT & $\begin{array}{c}\text { Demanda interna } \\
\text { (Secretaria de } \\
\text { Ciência, Tecnologia } \\
\text { e Insumos } \\
\text { Estratégicos/MS) }\end{array}$ & Sim & $\begin{array}{c}\text { Neoplasias } \\
\text { [tumores] (C00- } \\
\text { D48) }\end{array}$ & 2018 \\
\hline $\begin{array}{c}\text { Diretrizes } \\
\text { Diagnósticas e } \\
\text { Terapêuticas do } \\
\text { Carcinoma de Mama }\end{array}$ & $\begin{array}{c}\text { Carcinoma de } \\
\text { Mama }\end{array}$ & Incorporar & PCDT & $\begin{array}{l}\text { Demanda interna } \\
\text { (Secretaria de } \\
\text { Ciência, Tecnologia } \\
\text { e Insumos } \\
\text { Estratégicos/MS) }\end{array}$ & Nẵo & $\begin{array}{c}\text { Neoplasias } \\
\text { [tumores] (C00- } \\
\text { D48) }\end{array}$ & 2019 \\
\hline
\end{tabular}

Fonte: Elaborado pelo próprio autor baseada nos dados extraídos da CONITEC. 
Observou-se que as últimas incorporações de medicamentos para esta patologia foram realizadas em 2017, com a inclusão de Trastuzumabe e Pertuzumabe. Após este período, apenas foram sugeridas incorporações de protocolos clínicos para o câncer de mama.

\section{Discussão}

A incidência dos casos de câncer tornou evidente problemas de saúde pública com destacada relevância epidemiológica em todo o mundo. O crescimento dos gastos com saúde vem preocupando cada vez mais os gestores e em especial os dos países com sistemas universais como o brasileiro, por ser mais comprometido com aumento do número de casos e necessidade de incorporação de novas tecnologias, o que eleva significativamente os custos para diagnóstico e tratamentos, e dificulta ainda mais o acesso às terapias (Meropol, 2009).

Segundo Oliveira \& Soares, (2020), em um estudo proposto com o intuito de levantar a situação no atendimento de pacientes diagnosticados com câncer de mama em um referido município da Bahia, entre os problemas relacionados destacamse a vulnerabilidade na sistemática de integração na rede de atenção, uma rede de suporte para diagnóstico com um difícil acesso e principalmente um financiamento incondizente com a realidade. É uniforme, dentro deste estudo levantado, a clareza quanto ao subfinaciamento do SUS, sendo descrito como surreal, e inviabilizando a administração dos procedimentos, que mesmo garantindo a assistência mínima assegurada pela Emenda Constitucional (EC) No. 29 de 2000, a EC No.95 de 2016 limita o acesso através do congelamento dos gastos públicos federais em saúde, ocasionando o insucesso na prestação do atendimento (Oliveira \& Soares, 2020).

Uma outra problemática encontrada é a incorporação de novas tecnologias, eventualmente relacionada a judicialização para a aquisição das novas tecnologias já disponível para o tratamento com um melhor prognóstico. Este impasse se dá normalmente quando os preços praticados no mercado são incondizentes com os aplicáveis ao orçamento público, cabendo a população a busca pelos seus direitos na justiça (Instituto Nacional do Câncer, 2021).

As questões que envolvem o financiamento do SUS são históricas e permanece como preocupação para os seus gestores. A promessa de garantir a universalidade e a integralidade da assistência, em uma realidade marcada por restrições orçamentárias e financeiras, distribuir recursos de forma equânime, num País de tantas desigualdades sociais e regionais, tem sido um problema frequente (Brasil, 2011a).

Ocorre que muitas entidades desconhecem os custos reais dos procedimentos realizados, ao passo que não possuem controle fidedigno de seus gastos, ocasionando dúvidas quanto à suficiência destes repasses na cobertura dos custos efetivamente incorridos. A este cenário soma-se o fato de que o aumento dos gastos públicos com produtos para a saúde cresce em função da incorporação de tecnologias mais modernas e novos medicamentos (Medici, 2001; Barnum \& Kutzin, 1993).

É aceitável até certo ponto despender mais recursos com um produto ou medicamento mais eficaz, porém, uma avaliação criteriosa deve ser considerada. Avaliar aspectos como custo-efetividade das tecnologias de saúde e prioridades dos gastos é sem dúvida essencial para qualquer debate que se proponha democrático na área da saúde. Um estudo mostrou que no ano de 2018 foi registrado o maior número de tecnologias incorporadas pelo SUS. Não obstante, faltaram registros na literatura para justificar tal aumento nos gastos públicos com a saúde. Em paralelo, foi observado que os PCDTs daquele ano apresentaram inclusão massiva de recomendações de incorporações, sendo os medicamentos, a tecnologia mais incorporada. Ademais, percebeu-se que a indústria farmacêutica teve participação relevante como demandante externo na avaliação das tecnologias (Rodrigues-Filho \& Pereira, 2021). Isso demonstra que os gestores das Instituições Públicas como Hospitais, por exemplo, apresentam um papel pouco presente na demanda de incorporações de novas tecnologias, incluindo novas alternativas terapêuticas. 
Os resultados do presente trabalho mostraram que há uma estagnação no montante repassado, como observado na Figura 3, a qual traz os custos com quimioterápicos de acordo com a Tabela SUS. No entanto, a incorporação de novas tecnologias no SUS para o tratamento do câncer se torna praticamente inviabilizada devido à fixação dos valores dos tratamentos quimioterápicos, que em sua maioria são elevados. Isso força a utilização de produtos já consolidados e restringe o acesso a tecnologias que poderiam proporcionar uma maior adesão ao tratamento ou ainda uma melhor qualidade de vida do paciente.

É inegável que à medida que a abordagem da terapia alvo evoluiu para o câncer de mama, os tratamentos sistêmicos e adjuvantes também evoluíram. Pesquisas na área demonstraram que a quimioterapia adjuvante, hormonioterapia e terapiasalvo anti-HER2 reduzem os riscos de recidiva e melhoram a sobrevida global. O primeiro anticorpo a ser aplicado com sucesso no câncer de mama foi o trastuzumabe, e hoje é considerado um dos medicamentos essenciais para o combate a esta neoplasia. A experiência de incorporação do trastuzumabe no SUS foi bastante exitosa, porém o custo para sua aquisição durante a vigência da patente comprometeu em $75 \%$ dos recursos dos oncológicos dentre os 10 mais onerosos, como observado por Castro em 2020, quando avaliou a trajetória da queda da patente do trastuzumabe (Castro, 2020).

Neste estudo, foi observado que apesar de o IMIP, estabelecimento pertencente à Rede Oncológica de Pernambuco, possuir o segundo maior percentual no atendimento de quimioterapias, este é um dos estabelecimentos que possui uma média evolutiva decrescente ao longo dos anos $(-1,54 \%)$, ou seja, vem realizando menos procedimentos quimioterápicos entre os anos de 2015 e 2020. Isso pode indicar a dificuldade na gestão dos recursos recebidos frente à incorporação de novas tecnologias, sendo necessário em alguns casos, reduzir a quantidade de procedimentos realizados para compensar o aumento no valor da tecnologia utilizada. Para corroborar, o estudo realizado por Oliveira \& Soares (2020), que avaliou o acesso de mulheres com câncer de mama aos serviços de atenção à saúde, apontou financiamento insuficiente e fragilidade nos mecanismos de integração da rede de atenção, ocasionando rastreamento mamográfico ineficaz e dificuldade de acesso aos exames e procedimentos especializados. Tais achados podem interferir no prognóstico da doença e na qualidade de vida das pacientes (Oliveira \& Soares, 2020).

As opções para o tratamento de neoplasias através dos quimioterápicos são extensas e frequentemente há o lançamento de novos medicamentos pela indústria farmacêutica, possuindo em sua grande maioria, um valor muito significativo. Os tratamentos são pagos mensalmente através de valores fixos, sem considerar as individualidades de cada paciente, definido pelo Ministério da Saúde. Os valores não detalham quais os produtos devem ser adquiridos, cabendo às unidades credenciadas a seleção dos produtos, incluindo na sua padronização e definindo protocolos clínicos, quando necessário (Brasil, 2013).

Por essas questões, os gestores do SUS estão cientes que a inclusão na lista de padronizados não garante acessibilidade absoluta, ou seja, que o medicamento necessário esteja disponível na quantidade necessária para o paciente. Diferentes razões podem provocar situações de falta, porém, algumas estratégias são usadas rotineiramente nas centrais de quimioterapias durante o preparo destas, como o compartilhamento de frascos e o agendamento dos pacientes de acordo com a terapia a ser utilizada, para superar parte das falhas de fornecimento regular dos quimioterápicos, evitando desfechos desfavoráveis com o paciente, (Castro, 2020).

Foi observado que a quimioterapia prévia para o tratamento do câncer de mama possui um custo total, calculado pelo custo individual versus a quantidade de procedimento realizada no período, maior que a quimioterapia do câncer de mama avançado, denominado tratamento de $2^{\mathrm{a}}$ linha, que é mais oneroso individualmente. Isso pode indicar que em Pernambuco o diagnóstico precoce com o rastreio tem funcionado na Atenção Básica, que é um espaço privilegiado para ações de detecção precoce de câncer, ações essas que, comprovadamente, terão impacto na sobrevida do paciente em muitos tipos de câncer. 
Estudos como estes são importantes para nortear os gestores públicos na análise de eficiência das políticas de rastreio dos diferentes tumores.

Após o levantamento dos custos com os tratamentos adjuvantes e neoadjuvantes utilizados para o combate ao câncer de mama, em um hospital referência de Pernambuco, foi possível observar que a receita prevista é muito superior aos valores financiados pelo SUS. Com os resultados encontrados, apenas é possível prever que o cenário se torna ainda pior ao considerar a projeção dos gastos com essas quimioterapias nas próximas décadas, levando em conta ainda que o financiamento destes esteja congelado, se forem mantidas as proporções observadas nos últimos cinco anos.

Para corroborar com estes achados, uma análise realizada em 2006 sobre os custos diretos com esquemas terapêuticos para o tratamento oncológico de pacientes pediátricos, comparando com as receitas obtidas, apontou que o esquema terapêutico para quimioterapia utilizado para o carcinoma de suprarrenal apresentava custo elevado, que ultrapassava o valor previsto na APAC. Além disso, o tratamento quimioterápico para o glioma com a utilização de uma inovação terapêutica (temozolamida), na época, apresentava custo 3,18 vezes maior que o valor estabelecido para a APAC (Lavoyer, 2006). O custo médio por sessão de quimioterapia para tratamento de câncer de mama em mulheres em um hospital privado ficou em $\mathrm{R} \$$ 1.783,01. Deste, os medicamentos representaram 93,75\% (R \$ 1671,66) do custo total, o que chama atenção para necessidade da otimização das despesas sem prejuízos ao atendimento aos pacientes (Nóbrega, 2014).

Um dos motivos que pode provocar a redução dos procedimentos quimioterápicos, como dito anteriormente, é o financiamento insuficiente ou desatualizado. Diante da análise dos valores recebidos por procedimento quimioterápico, se percebeu que não houve, durante o período observado, nenhum ajuste monetário ao longo dos anos nos tratamentos quimioterápicos atendidos nos estabelecimentos de saúde de Pernambuco observados. Isso indica que a atual forma de terapia oncológica para o câncer de mama no estado não tem acompanhado as devidas incorporações frequentemente recomendadas nos PCDTs e que são preconizadas e aprovadas por Agências internacionais.

Para o tratamento do câncer de mama, por exemplo, diversos são as novas tecnologias. No ano de 2017, houve a regulamentação pela Agência Nacional da Vigilância Sanitária (ANVISA) do medicamento antineoplásico Atezolizumabe, conhecido comercialmente como Tecentriq. Este suprime a atividade citotóxica das células $\mathrm{T}$ no microambiente tumoral, inibindo a resposta imune, sendo um anticorpo monoclonal de imunoblobulina G1 (IgG1), se ligando diretamente ao PDL-1, ligante de morte programada e promovendo um duplo bloqueio nos receptores PD-1 e B7.1. A indicação deste no tratamento de câncer de mama deve ser associado ao nab-paclitaxiel, podendo ser administrado em pacientes adultos cujo câncer seja diagnosticado como triplo negativo localmente ressecável ou metastático que apresentem o PD-L1 $\geq 1 \%$, e nunca antes tratado quimioterapicamente para doenças metastáticas. A utilização deste pode aumentar a sobrevida do paciente e um maior tempo sem o agravamento da doença (Brasil, 2017; European Medicines Agency, 2021a).

Outro exemplo é o inibidor de proteína quinase, Abemaciclibe, conhecido comercialmente como Verzenios, e regulamentado pela ANVISA em 2019. Este impede a fosforilação retinoblastoma (Rb), inibindo no ciclo celular da fase $\mathrm{S}$ a progressão de G1 e suprimindo o crescimento tumoral, já que a ciclina D1/CDK4 viabiliza a fosforilação do Rb, o aumento celular e o crescimento tumoral. Este medicamento é indicado para o câncer de mama precoce associado a terapias endócrinas, com agravo de risco de reincidência, receptor hormonal (HR) positivado, receptor de crescimento epidérmico humano 2 (HER2) negativado e linfonodos positivos, assim como para o avançado ou metastático, HR positivado e HER2 negativado. Pôde-se concluir que o medicamento aumenta o tempo de agravamento da doença, assim como prolonga o tempo de sobrevivência dos pacientes (European Medicines Agency, 2021b).

Apesar de ambos os medicamentos comprovarem um aumento no tempo relacionado ao agravamento da doença e um prolongamento do tempo de sobrevivência em paciente de câncer de mama quando comparado com os tratamentos atualmente utilizados, ambos ainda não foram incorporados nas Diretrizes Diagnósticas e Terapêuticas do câncer de pulmão (MS, 2019). 
Em paralelo, sabe-se que outros fatores permitem a incorporação de algumas tecnologias, como a quebra da patente de alguns quimioterápicos, a saber, o Tamoxifeno e o Rituximab, favoreceu a sobrevivência econômica de alguns esquemas quimioterápicos no País, pois a redução do custo compensou a falta de reajuste perante o aumento da inflação. No entanto, após extensa busca na literatura, não foi possível identificar como são feitos os reajustes dos valores repassados por tratamentos quimioterápicos.

O financiamento para ações e serviços em saúde no Brasil vem oscilando ao longo dos anos próximo dos 8\% (oito por cento) do Produto Interno Bruto, ficando 2 a 3 pontos percentuais abaixo dos de países desenvolvidos (Canadá e Reino Unido) com sistemas de saúde universais. Considerando que o PIB brasileiro em 2020 foi de aproximadamente 6,8 trilhões de reais podemos estimar que foram investidos em saúde mais de $\mathrm{R} \$ 544$ bilhões pelos setores privados e públicos, sendo os entes federativos responsável por cerca de 45\% (220 bilhões de reais) deste montante investido em saúde pública (Figueiredo, 2018).

Os valores diretos e indiretos relacionados às sessões de quimioterapia devem ser integralmente financiados. Para cada tipo de câncer é preconizado um montante fixo mensal, cabendo às unidades de saúde implantar ações que minimizem os custos para que se adequem aos valores previamente estabelecidos, reportando os custos ao MS que após análise efetuará o pagamento dos aprovados (Brasil, 2007). O modelo de pagamento utilizado atualmente para o tratamento oncológico é feito através de pacote. Este modelo deveria ser um bom método, do ponto de vista institucional, pois é factível financeiramente. Porém, alguns pacientes podem não receber o melhor tratamento para sua doença. Afinal, para oferecer o tratamento mais adequado possível, existe a necessidade de implantar protocolos clínicos bem estabelecidos, definir a melhor tecnologia a ser incorporada e atualizar constantemente os valores dos pacotes, o que não tem acontece.

Ocorre que o orçamento para saúde no Brasil tem sofrido constantes pressões para contenção, incluindo mais recentemente os efeitos impostos pela emenda constitucional 95 que congela os orçamentos da União por 20 anos, o que aumenta significativamente o risco de restrição do acesso aos serviços de saúde e tratamentos (Brasil, 2016).

Cabe ainda ressaltar que os procedimentos quimioterápicos pertencem ao grupo de média e alta complexidade, que limita a quantidade de procedimentos disponibilizados por meio das pactuações estabelecidas pelos Gestores do SUS, ou seja, é estabelecido um limite de gastos mediante do montante financeiro máximo repassado, devendo os procedimentos totais se limitar a esta quantia. Caso os valores ultrapassem este total, deverá ser ajustado nos anos seguintes, ou deverá ser realizada a revisão orçamentária por meio de debates técnicos, sendo um processo moroso.

Neste contexto, os gestores públicos necessitam avaliar melhor a real situação de aporte financeiro disponibilizado ao SUS para poder acompanhar as novas tecnologias que poderiam ser incorporadas. Não é uma situação simples e exigirá esforços conjuntos, tanto das Esferas Federais, quanto Estaduais e Locais para melhor gerência dos recursos voltados para o tratamento de pacientes oncológicos.

\section{Considerações Finais}

O aumento gradativo da incidência e mortalidade pelo câncer, somados ao crescimento da expectativa de vida e aos valores engessados do financiamento dos tratamentos oncológicos, nos últimos anos, deflagra um problema importante para os gestores de saúde. Através da análise dos dados extraídos não se pode visualizar critérios para o estabelecimento dos valores que não foram ajustados de acordo com os anos em contraponto ao aumento anual dos medicamentos e da inflação, fatores que influenciam diretamente no aumento dos custos dos tratamentos quimioterápicos. Portanto, estudos como estes são importantes para melhor embasamento dos gestores públicos na tomada de decisão referente aos gastos em saúde.

Foi possível também visualizar que a padronização dos procedimentos, e o engessamento monetário dos tratamentos, podem ser potencialmente danosos, pois impossibilitam a aquisição de novas tecnologias, ou novos produtos que possam 
melhorar a qualidade de vida, aumentar a sobrevida ou ainda facilitar a adesão do paciente ao tratamento. Os fatos observados têm sido gatilhos para dificultar a garantia da população ao acesso pleno do sistema público de saúde no Brasil, no que se refere ao diagnóstico e tratamento da doença. Dessa forma, a atual forma de financiamento do tratamento quimioterápico para o câncer de mama não se mostrou financeiramente sustentável no Sistema Único de Saúde.

\section{Referências}

Amorim, A. S. R. Sustentabilidade: entre a utopia, a prática e a estratégia empresarial. Dissertação (Mestrado Profissional em Administração) ESAG/ Escola Superior de Administração e Gerência. Universidade do Estado de Santa Catariana, Florianópolis.

Barnum, H.; Kutzin, J. (1993). Public Hospitals in Developing Countries: Resource use, cost, financing. Washington: The World Bank.

Barrios, C. H.; Amorim, G.; Tavares, M.; Sahade, M. C. M.; Bedin, S. R.; Reinert, T. (2021). Diretrizes De Tratamentos Oncológicos Mama: Estadiamento. Diretrizes De Tratamentos Oncológicos Recomendados Pela Sociedade Brasileira De Oncologia Clínica. Sociedade Brasil De Oncologia<25.-DiretrizesSBOC-2021---Mama-estadiamento-2.pdf>.

Brasil. (2005). Portaria GM MS no 2.439 de 8 de dezembro de 2005, Institui a Política Nacional de Atenção Oncológica: Promoção, Prevenção, Diagnóstico, Tratamento, Reabilitação e Cuidados Paliativos, a ser implantada em todas as unidades federadas, respeitadas as competências das três esferas de gestão de 8 de dezembro de 2005 .

Brasil. (1990). Lei 8.142, de 28 de dezembro de 1990. Dispõe sobre a participação da comunidade na gestão do Sistema Único de Saúde (SUS) e sobre as transferências intergovernamentais de recursos financeiros na área da saúde e dá providências.<http://www.planalto.gov.br/ccivil_03/leis/L8142.htm.>.

Brasil. (1993). Lei n 8.666, de 21 de junho de 1993< http://www.planalto.gov.br/ccivil_03/leis/18666cons.htm>.

Brasil. (2016). Emenda Constitucional 95, de 15 de dezembro de 2016. Altera o Ato das Disposições Constitucionais Transitórias, para instituir o Novo Regime Fiscal, e dá outras providências.

Brasil. (2017). Ministério da Saúde. Gabinete do Ministro. Anexo IX da Portaria de consolidação n. ${ }^{\circ} 2$, de 28 de setembro de 2017. Dispõe sobre a Política Nacional para Prevenção e Controle do Câncer. Diário Oficial da República Federativa do Brasil, Poder Executivo, Brasília, DF.

Brasil. (2020). ABC Do Câncer: Abordagens Básicas Para O Controle Do Câncer. Instituto Nacional De Câncer José Alencar Gomes Da Silva; Organização Mario Jorge Sobreira Da Silva. - 4. Ed. Rev. Atual. - Rio De Janeiro: Inca,. Ministério da Saúde.

Brasil. Constituição (1988). Constituição da República Federativa do Brasil. Brasília, DF: Centro Gráfico.

Brasil. (1990). Lei 8.080/90, de 19 de setembro de 1990. Dispõe sobre as condições para promoção, proteção e recuperação da saúde, a organização e o funcionamento dos serviços correspondentes e dá outras providências <http://www.planalto.gov.br/ccivil_03/leis/18080.htm>.

Brasil. (2001). Ministério da Saúde. Secretaria de Políticas de Saúde. Departamento de Atenção Básica. Gerência Técnica de Assistência Farmacêutica. Assistência farmacêutica: instruções técnicas para a sua organização. 114 -14.

Brasil. (2014). Portaria $n^{\circ}$ 140, de 27 de fevereiro de 2014-Redefine os critérios e parâmetros para organização, planejamento, monitoramento, controle e avaliação dos estabelecimentos de saúde habilitados na atenção especializada em oncologia e define as condições estruturais, de funcionamento e de recursos humanos para a habilitação destes estabelecimentos no âmbito do Sistema Único de Saúde (SUS).<https://bvsms.saude.gov.br/bvs/saudelegis/sas/2014/prt0140_27_02_2014>.

Brasil. (2016). Portaria $N^{o}$ 1.354, De 4 De Outubro De 2016. Ministério da Saúde. <https://www.in.gov.br/materia//asset_publisher/Kujrw0TZC2Mb/content/id/24340900>.

Brasil. (2018). Portaria Conjunta $n^{\circ}$ 04, de 23 de janeiro de 2018. Aprova as Diretrizes Diagnósticas e Terapêuticas do Carcinoma de Mama Ministério da Saúde. Secretaria de Atenção à Saúde. Secretaria de Ciência, Tecnologia e Insumos Estratégicos.

Brasil. (2019). Portaria saes n. 1.399, de 17 de dezembro de 2019. Redefine os critérios e parâmetros referenciais para a habilitação de estabelecimentos de saúde na alta complexidade em oncologia no âmbito do SUS.

Brasil. (2021). Agência Nacional de Vigilância Sanitária. Consultas / Medicamentos / Tecentriq. <https://consultas.anvisa.gov.br/\#/medicamentos/25351102551201639/>.

Brasil. (2021). Decreto $n^{\circ}$ 7.507, de 27 de junho de 2011.Dispõe sobre a movimentação de recursos federais transferidos a Estados, Distrito Federal e Municípios, em decorrência das leis citadas. .<http://www.planalto.gov.br/ccivil_03/_Ato2011-2014/2011/decreto/d7507.htm>.

Brasil. (2021). Manual De Bases Técnicas Da Oncologia - Sia/Sus - Sistema De Informações Ambulatoriais. Ministério Da Saúde/ Secretaria De Atenção À Saúde/ Departamento De Regulação, Avaliação E Controle/Coordenação-Geral De Gestão Dos Sistemas De Informações Em Saúde - $28^{a}$ Edição.

Brasil. Ministério da Saúde. Instituto Nacional de Câncer (INCA). Estimativa 2020: A incidência de câncer no Brasil. Rio de Janeiro: 120.

Bray, F., Ferlay, J., Soerjomataram, I., Siegel, R. L., Torre, L. A., \& Jemal, A, (2018). Global cancer statistics 2018: GLOBOCAN estimates of incidence and mortality worldwide for 36 cancers in 185 countries. CA: a cancer journal for clinicians, 68(6), 394-424. 
Research, Society and Development, v. 10, n. 16, e459101623883, 2021 (CC BY 4.0) | ISSN 2525-3409 | DOI: http://dx.doi.org/10.33448/rsd-v10i16.23883

Castro, A. M. A. (2020). O impacto de políticas públicas no acesso ao trastuzumabe para o tratamento de pacientes com câncer de mama em um Hospital Universitário.Dissertação de mestrado. Faculdade de Medicina da Universidade de São Paulo: Gestão em Organização de Saúde.

Chene, G., Lamblin, G., Le Bail-Carval, K., Beaufils, E., Chabert, P., Gaucherand, P., ... \& Coppens, Y. (2016). Le (s) cancer (s) de Lucy: une origine préhistorique?. Gynécologie Obstétrique \& Fertilité, 44(12), 690-700.

Coleman, Cath,y.(2017). Early detection and screening for breast cancer. In: Seminars in oncology nursing. WB Saunders,141-155.

Diário Oficial da União. (2013). Portaria No 874, DE 16 DE MAIO DE 2013, Institui a Política Nacional para a Prevenção e Controle do Câncer na Rede de Atenção à Saúde das Pessoas com Doenças Crônicas no âmbito do Sistema Único de Saúde (SUS). Diário Oficial da União.

European Medicines Agency. (2021). Tecentriq (atezolizumab): Um resumo sobre Tecentriq e porque está autorizado na $E U<$.https://www.ema.europa.eu/documents/overview/tecentriq-epar-medicine-overview_pt.pdf.>.

European Medicines Agency. (2021). Verzenios (abemaciclib) Um resumo sobre Verzenios e porque está autorizado na UE <https://www.ema.europa.eu/documents/overview/verzenios-epar-medicine-overview_pt.pdf>.

Fachini, C. (2005). Sustentabilidade financeira e custos de transação em uma organização de microcrédito no Brasil. Piracicaba: ESALQ/USP.

Figueiredo, J. O., Prado, N. M. D. B. L., Medina, M. G., \& Paim, J. S. (2018). Gastos público e privado com saúde no Brasil e países selecionados. Saúde em Debate, 42, 37-47.

Instituto Nacional do Câncer (INCA). (2021). Oncologia no SUS: os caminhos do financiamento

Lavoyer, F. C. G .(2006). Análises parciais de custos diretos nos esquemas terapêuticos oncológicos em pediatria. Curitiba. 95 Biblioteca Virtual em Saúde.

Médici, A. C. (2001). Hospitais universitários: passado, presente e futuro. Revista da Associação Médica Brasileira, 47, 149-156.

Meropol, N. J., Schrag, D., Smith, T. J., Mulvey, T. M., Langdon Jr, R. M., Blum, D., Ubel, P.A., Lowell, E., \& Schnipper, L. E.(2009). American Society of Clinical Oncology guidance statement: the cost of cancer care. Journal of Clinical Oncology, 27(23), 3868-3874.

Ministério da Saúde (MS). (2019). Secretaria De Atenção À Saúde Secretaria De Ciência, Tecnologia E Insumos Estratégicos. Portaria Conjunta Nº 5 , DE 18 DE ABRIL DE 2019.

Nòbrega, C. R \& Lima, A. F. C. (2014). Custo de procedimentos relacionados ao tratamento quimioterápico ambulatorial de mulheres portadoras de câncer de mama. Rev Esc Enferm USP. 48(4):698-705.

Oliveira, M. A. Bermudez, J. A. Z.; Osorio-De-Castro, C. G. S. (2007). Assistência farmacêutica e acesso a medicamentos. Rio de Janeiro: Editora Fiocruz.

Oliveira, S. B., \& Soares, D. A. (2020). Acesso ao cuidado do câncer de mama em um município baiano: perspectiva de usuárias, trabalhadores e gestores. Saúde em Debate, 44, 169-181.

Rodrigues Filho, F. J., \& Pereira, M. C. (2021). O perfil das tecnologias em saúde incorporadas no SUS de 2012 a 2019 : quem são os principais demandantes? Saúde em Debate, 45, 707-719.

Silva, M. J. S., O’Dwyer, G., \& Osorio-de-Castro, C. G. S. (2019). Cancer care in Brazil: structure and geographical distribution. BMC cancer, 19(1), 1-11.

Vieira, F. S. (2020). O financiamento da saúde no Brasil e as metas da Agenda 2030: alto risco de insucesso. Revista de Saúde Pública, 54.

World Health Organization. (2021). Global Cancer Observatory.

World Health Organization. (2021). Guidelines for the early detection and screening of breast cancer. 